\title{
Evaluation of Disturbance Propagation through Heat Exchanger Networks
}

\author{
M. M. Gouda \\ Chemical and Petroleum Process \\ Engineer at Qarun Petroleum \\ Company
}

\author{
M. Kaoud \\ Chemical and Petroleum Process \\ Engineer at Khalda Petroleum \\ Company
}

\author{
S. M. Aly \\ Professor Chemical and Petroleum \\ Refining Engineering Department \\ Suez University
}

\begin{abstract}
For a given heat exchanger network it is often necessary to determine the behavior of disturbances in the target temperature when inlet flow rate variations, and what is the ability or flexibility of the heat exchangers network to meet the design requirement at new operating conditions. This paper introduced a model to evaluate the disturbance propagation through a heat exchanger network without bypass, each exchanger in the network is represented by a set of algebraic equation with neglect high order differentiation terms. The model can be used to estimate the maximum deviation of system outputs when it experiences the worst combination of various types of disturbances, the model is developed for the analysis of controllability rather than flexibility, during the process design stage. The model is illustrated cause \& effect relationship between the target temperatures and the disturbances in inlet temperatures and heat capacity flow rates. - The method has been tested for two problems reported in the literature.
\end{abstract}

\section{Keywords}

Heat exchanger networks, disturbance, flexibility, controllability.

\section{INTRODUCTION}

The determination of the steady state response of heat exchanger networks to imposed disturbances can be solved using different approaches. The most traditional methodologies involve the simultaneous solution of a set of algebraic equations. Other approaches have also been developed recently, but these methods require significant experience of the engineer in order to identify any feedback loops from the grid diagram and this can be very difficult in complex networks [1]. Perhaps the most feasible approach to the solution of the problem by hand is the derivation of response equations [1]. In this method, response equations have been derived that facilitate the calculation of disturbances as they propagate through the network. For this, a route through the network must be established. A special treatment is given for the solution when a feedback loop is found in the network. The introduced response equations allow the prediction of the change of the outlet stream temperatures, but there are no equations to predict changes for disturbances occurring in all system variables. A feedback factor must be calculated, which is not easy to obtain, especially for complex and large networks. Probably, the earliest work on disturbance propagation analysis for process synthesis was pioneered by Kotjabasakis and Linnhoff [2]. They introduced a procedure for considering flexibility right at the design stage and established a trade-off between flexibility and total cost of a heat exchanger network (HEN). Their procedure makes extensive use of what they termed sensitivity tables; thus, it is inefficient to the analysis of a complex HEN. Ratnam and
Patwardhan [3] developed a methodology based on the LMTD design method proposed in the textbook by Kern [4]. In this, if any, change is introduced into the network, all the target temperatures are evaluated again by solving a set of equations. This model does not provide an explicit relationship between disturbances and target temperatures. Li et al. [5] developed linear equations for modeling the disturbance propagation in a HEN. Their model is also for process flexibility analysis. Li and co-workers introduced an average temperature difference to approximate a logarithmic mean temperature difference and derived approximate linear relationships between source and target temperature disturbances. Unfortunately, their model completely ignores the cross effect of the disturbances of temperatures and those of heat capacity flowrates. This leads to considerable computational errors when both types of disturbances exist. In addition, their model relies on the simulation of a given HEN structure when flow rate variations are considered. Through simulation, piecewise linearization for each temperature concerned can be obtained. This is very cumbersome when a number of process alternatives are to be analyzed. Yang et al [6] described a simplified system model for rapidly evaluating the disturbance propagation through a heat exchanger network. The model depicts cause-effect relationships between a set of stream output variables (target temperature fluctuations) and a set of input variables (disturbances of source temperatures and heat capacity flow rates). The model, which consists of a set of linear equations of disturbances, can precisely evaluate the propagation caused by even severe temperature disturbances and/or by moderate heat capacity flow rate fluctuations. This model produces acceptable results within narrow ranges of values of disturbances, but will lead to considerable errors, especially when high mass- flow disturbances occur. This procedure has limited use due to the simplifying assumptions applied and can only handle heat exchanger configurations with truly counter-flow. P. J. Heggs and F. Vizcaino [7] developed a method to evaluate the propagation of steady state disturbances through heat exchanger networks. Each exchanger in the network is represented algebraically using the effectiveness-NTU methodology. The properties of the resulting structural disturbance matrices for the network play a vital role in this model. Most industrial processes operate under uncertain operating conditions. These processes require flexible operation and this can be achieved by using an adequate heat exchanger network. A systematic method is developed to evaluate the propagation of disturbances through heat exchanger networks. 


\section{DISTURBANCE PROPAGATION MODELING}

A disturbance propagation model was adopted from Yang et al. modeling approach [6] and the model is explained below.

A disturbance propagation model for unit heat exchanger is as follows:-

$\delta T^{t}=D t \delta T^{s}+D m \delta M C p$

where:

$\left(\begin{array}{l}\delta T_{h}^{t} \\ \delta T_{c}^{t}\end{array}\right)$

$=\left(\begin{array}{cc}1-\alpha & \alpha \\ \beta & 1-\beta\end{array}\right)\left(\begin{array}{l}\delta T_{h}^{t} \\ \delta T_{c}^{t}\end{array}\right)$

$+\left(\begin{array}{cc}\alpha_{h}(2-\alpha) & -\alpha \alpha_{c} \\ \alpha_{h} \beta & -\alpha_{c}(2-\beta)\end{array}\right)\left(\begin{array}{l}\delta M C p_{h} \\ \delta M C p_{c}\end{array}\right)$

$\alpha=\frac{T_{h}^{s}-T_{h}^{t}}{T_{h}^{s}-T_{c}^{s}}$

$\beta=\frac{T_{c}^{t}-T_{c}^{s}}{T_{h}^{s}-T_{c}^{s}}$

$\alpha_{h}=\frac{\Delta T_{h}}{2 M C p_{h}}$

$\alpha_{c}=\frac{\Delta T_{c}}{2 M C p_{c}}$

Where $T$ and $\delta T$ are the stream temperature and temperature fluctuation respectively. $M c p$ and $\delta M c p$ are the heat capacity flow rate and heat capacity flow rate fluctuation respectively. Superscripts $\mathrm{s}$ and $\mathrm{t}$ refer to source and target respectively subscripts $h$ and $c$ refer to hot and cold respectively.

If a HEN contains $N_{e}$ heat exchangers, a system model can be obtained directly by lumping all units based models in the sequence of exchanger numbers that gives:-

$\delta T^{* \text { out }}=D_{t_{E i}} \delta T_{E_{i}}^{\text {in }}+D_{m_{E i}} \delta M c p^{*}$

$\delta T^{* \text { out }}=D t_{E}^{*} \delta T^{* \text { in }}+D m_{E}^{*} \delta M c p_{E}^{*}$

Where

$$
\begin{aligned}
& \delta T^{* \text { in }}=\left[\left(\delta T_{E_{1}}^{\text {in }}\right)^{T}\left(\delta T_{E_{2}}^{\text {in }}\right)^{T} \ldots\left(\delta T_{E_{N e}}^{\text {in }}\right)^{T}\right]^{T} \\
& \delta T^{* \text { out }}=\left[\left(\delta T_{E_{1}}^{\text {out }}\right)^{T}\left(\delta T_{E_{2}}^{\text {out }}\right)^{T} \ldots\left(\delta T_{E_{N e}}^{\text {out }}\right)^{T}\right]^{T} \\
& \delta M c p_{E}^{*}=\left[\left(\delta M c p_{E 1}\right)^{T}\left(\delta M c p_{E 1}\right)^{T} \ldots\left(\delta M c p_{E N_{e}}\right)^{T}\right]^{T} \\
& D_{t_{E}}^{*}=\operatorname{diag}\left\{D_{t_{E 1}}, D_{t_{E 2}} \ldots . D_{t_{E N_{e}}}\right\} \\
& D_{m_{E}}^{*}=\operatorname{diag}\left\{D_{m_{E 1}}, D_{m_{E 2}} \ldots . D_{m_{E N_{e}}}\right\}
\end{aligned}
$$

The following equation represents the system disturbance propagation model:

$\delta T^{t}=D_{t} * \delta T^{s}+D_{m} * \delta M C P$

Where

$D_{t}=D_{t 11}+D_{t 12} *\left(I-D_{t 22}\right)^{-1} * D_{t 21}$

$D_{m}=D_{m 1}+D_{t 12} *\left(I-D_{t 22}\right)^{-1} * D_{m 2}$

Where

$D_{t}^{*}=\left(\begin{array}{cc}D_{t 11} & D_{t 12} \\ D_{t 21} & D_{t 22}\end{array}\right)=V 1 * D_{t_{E}}^{*} * V 2$
$D_{m}^{*}=\left(\begin{array}{c}D_{m 1} \\ D_{m 2}\end{array}\right)=V 1 * D_{m_{E}}^{*} * V 3$

Matrices $V 1, V 2$ and $V 3$ are system construction matrices.

\section{PROBLEM STATEMENT}

To control the target temperatures for a set of streams in a heat exchanger network the disturbance propagation due to any fluctuation in the inlet parameters in the heat exchangers network need to be evaluated to calculate the deviation in the target streams and construct a control scheme to eliminate that deviation. The interconnections among process streams, generate numerous downstream paths through which disturbances propagate. Some of these interconnections should be examined with extra care because intense disturbances might propagate through the paths generated by the interconnections, thereby detrimentally affecting the controllability of the process. This implies that the controllability of a process can be assessed by examining the disturbance propagation in a process structure. The following model evaluates the disturbance propagation in a HEN in two steps the first step is to evaluate a unit based disturbance propagation model the second step is to construct a system based disturbance model.

The following assumptions are applied in this work:-

1. No phase change occurs in any heat exchanger.

2. Constant heat capacity and constant over all heat transfer coefficient for each heat exchanger.

3. Changes in the streams pressure drop are negligible.

The data required are:

1. Heat exchanger network work flow

2. Hot and cold streams data for each unit heat exchanger i.e. (Inlet temperatures, outlet temperature and heat capacity flow rate for the streams) and the split ratio for any stream is required in case of splitting between multi-unit heat exchanger.

3. A specified range of uncertainties (Inlet temperature, heat capacity flow rates).

The goal here is to evaluate the disturbance propagation through the heat exchanger network and find the effect of changing an inlet parameter for any stream on the other streams even if they are not directly connected.

\section{CASE STUDY}

This is a 10 stream Heat exchanger network first studied by Ahmad [8], and Murty et al. [10] where they studied the most cost optimal design method which showed that the design of Murty et al. [10] have the optimal cost so in this work the disturbance propagation in this network will be checked.

Table 1. Input data for case study

\begin{tabular}{c|c|c|c} 
Stream & $\boldsymbol{T}_{\text {in }}\left({ }^{\circ} \mathrm{C}\right)$ & $\boldsymbol{T}_{\text {out }}\left({ }^{\circ} \mathrm{C}\right)$ & $\mathbf{M c p}\left(\boldsymbol{K} \boldsymbol{w}^{\circ} \boldsymbol{K}^{-\mathbf{1}}\right)$ \\
\hline H1 & 85 & 45 & 156.3 \\
\hline $\mathbf{H} 2$ & 120 & 40 & 50 \\
\hline H3 & 125 & 35 & 23.9 \\
\hline H4 & 56 & 46 & 1250 \\
\hline H5 & 90 & 86 & 1500 \\
\hline
\end{tabular}




\begin{tabular}{c|c|c|c|}
\hline H6 & 225 & 75 & 50 \\
\hline C1 & 40 & 55 & 466.7 \\
\hline C2 & 55 & 65 & 600 \\
\hline C3 & 65 & 165 & 180 \\
\hline C4 & 10 & 170 & 81.3 \\
\hline Hot utility & 200 & 198 & -- \\
\hline Cold utility & 15 & 25 & -- \\
\hline
\end{tabular}

Heat transfer coefficient $\mathrm{U}=0.025 \mathrm{KWm}^{2} \mathrm{~K}^{-1}$

Capital cost of heat exchanger $=60 * \mathrm{~A} \$ / \mathrm{m}^{2}\left(\mathrm{~A}=\right.$ area in $\left.\mathrm{m}^{2}\right)$

Cost of cold utility $=15 \$ \mathrm{KW}^{-1}$ year $^{-1}$

The following disturbances of uncertainties their effect on the streams target temperature need to be evaluated.

Table 2. Deviation in inlet parameters

\begin{tabular}{|c|c|c|}
\hline \multirow{2}{*}{ Stream } & \multicolumn{2}{|c|}{ Type of deviation } \\
\cline { 2 - 3 } & Inlet temp. & Heat capacity flow rate \\
\hline H1 & +5 & --- \\
\hline H2 & -10 & --- \\
\hline H3 & +5 & ---- \\
\hline C1 & ---- & $+2 \%$ \\
\hline
\end{tabular}

Cost of hot utility $=100 \$ \mathrm{KW}^{-1}$ year $^{-1}$

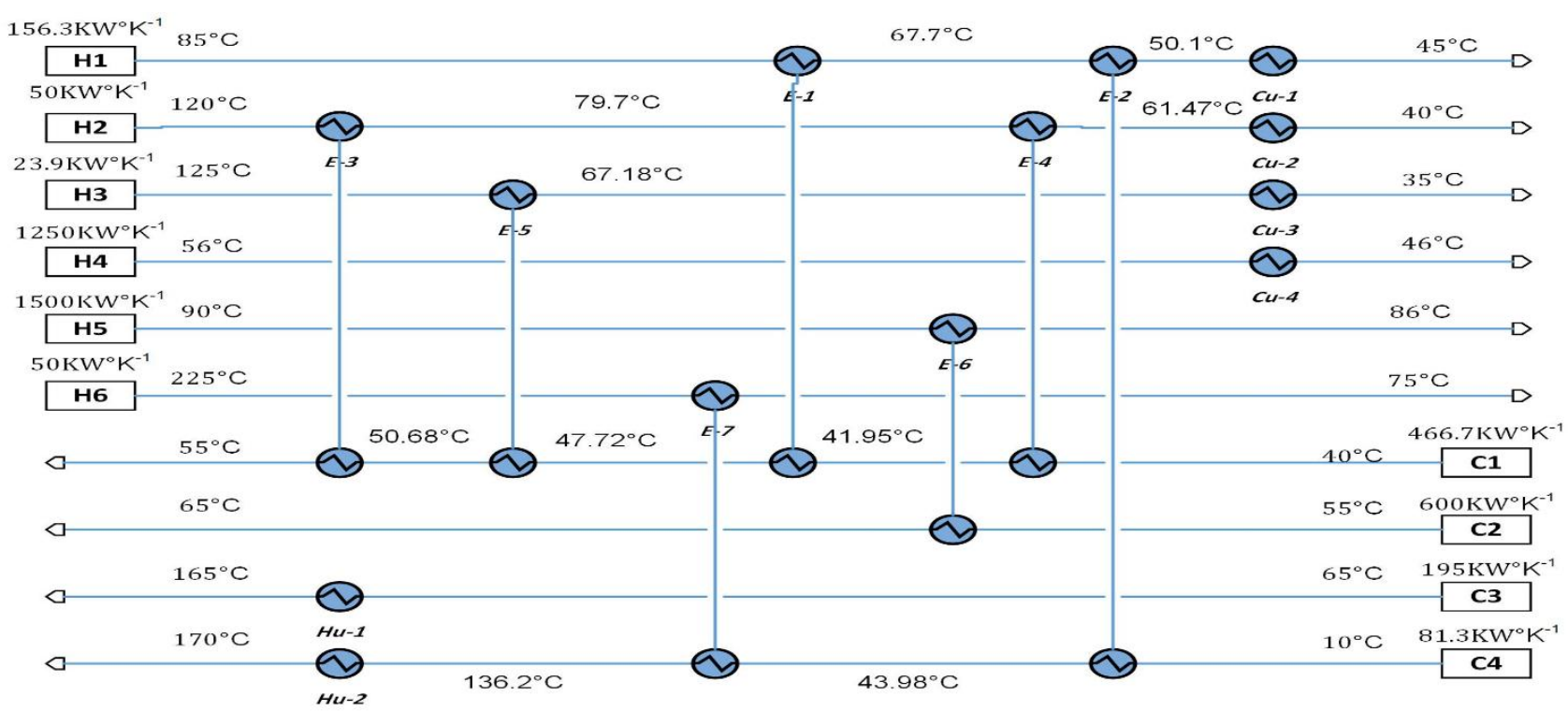

Figure 1.Heat exchanger network 10 streams

\subsection{Unit based model}

For each unit process heat exchanger the model in equation-1 is calculated using equations 2 through 6 to construct the model for the HEN in figure-1 is as follows:

Table 2. Input data for E-1

\begin{tabular}{|c|c|c|c|}
\hline Stream & $T_{\text {in }}\left({ }^{\circ} \mathrm{C}\right)$ & $T_{\text {out }}\left({ }^{\circ} \mathrm{C}\right)$ & $\mathrm{Mcp}\left(K w^{\circ} \mathrm{K}^{-1}\right)$ \\
\hline $\mathrm{H} 1$ & 85 & 67.777 & 56.3 \\
\hline $\mathrm{C} 1$ & 41.95 & 47.72 & 466.7 \\
\hline
\end{tabular}

$$
\begin{aligned}
{\left[\begin{array}{l}
\delta T_{m 1} \\
\delta T_{m 4}
\end{array}\right]=\left[\begin{array}{ll}
0.5999 & 0.4001 \\
0.1340 & 0.8660
\end{array}\right]\left[\begin{array}{l}
\delta T_{H 1}^{S} \\
\delta T_{m 5}
\end{array}\right] } & +\left[\begin{array}{ll}
0.0881 & -0.0025 \\
0.0074 & -0.0150
\end{array}\right]\left[\begin{array}{l}
\delta M c p_{H 1} \\
\delta M c p_{C 1}
\end{array}\right]
\end{aligned}
$$

Table 3. Input data for E-2

\begin{tabular}{|c|c|c|c|}
\hline Stream & $T_{\text {in }}\left({ }^{\circ} \mathrm{C}\right)$ & $T_{\text {out }}\left({ }^{\circ} \mathrm{C}\right)$ & $\mathrm{Mcp}\left(K w^{\circ} K^{-1}\right)$ \\
\hline $\mathrm{H} 1$ & 67.777 & 50.1 & 156.3 \\
\hline $\mathrm{C} 4$ & 10 & 43.98 & 81.3 \\
\hline
\end{tabular}

$$
\begin{aligned}
{\left[\begin{array}{l}
\delta T_{H 1}^{t} \\
\delta T_{m 6}
\end{array}\right]=\left[\begin{array}{ll}
0.6941 & 0.3059 \\
0.5882 & 0.4118
\end{array}\right]\left[\begin{array}{ll}
\delta T_{m 1} \\
\delta T_{C 4}^{s}
\end{array}\right] } \\
+\left[\begin{array}{ll}
0.0958 & -0.0639 \\
0.0332 & -0.2950
\end{array}\right]\left[\begin{array}{l}
\delta M c p_{H 1} \\
\delta M c p_{C 4}
\end{array}\right]
\end{aligned}
$$

Table 4. Input data for E-3

\begin{tabular}{|c|c|c|c|}
\hline Stream & $T_{\text {in }}\left({ }^{\circ} \mathrm{C}\right)$ & $T_{\text {out }}\left({ }^{\circ} \mathrm{C}\right)$ & $\mathrm{Mcp}\left(K w^{\circ} K^{-1}\right)$ \\
\hline $\mathrm{H} 2$ & 120 & 79.701 & 50 \\
\hline $\mathrm{C} 1$ & 50.683 & 55 & 466.7 \\
\hline
\end{tabular}

$$
\begin{aligned}
{\left[\begin{array}{l}
\delta T_{m 2} \\
\delta T_{C 1}^{t}
\end{array}\right]=\left[\begin{array}{ll}
0.4186 & 0.5814 \\
0.0623 & 0.9377
\end{array}\right]\left[\begin{array}{l}
\delta T_{H 2}^{s} \\
\delta T_{m 3}
\end{array}\right] } \\
+\left[\begin{array}{ll}
0.5717 & -0.0027 \\
0.0251 & -0.0090
\end{array}\right]\left[\begin{array}{l}
\delta M c p_{H 2} \\
\delta M c p_{C 1}
\end{array}\right]
\end{aligned}
$$

Table 5. Input data for E-4

\begin{tabular}{|c|c|c|c|}
\hline Stream & $T_{\text {in }}\left({ }^{\circ} \mathrm{C}\right)$ & $T_{\text {out }}\left({ }^{\circ} \mathrm{C}\right)$ & $\operatorname{Mcp}\left(K w^{\circ} K^{-1}\right)$ \\
\hline $\mathrm{H} 2$ & 79.701 & 61.47 & 50 \\
\hline $\mathrm{C} 1$ & 40 & 41.95 & 466.7 \\
\hline
\end{tabular}




$$
\begin{aligned}
{\left[\begin{array}{l}
\delta T_{H 2}^{t} \\
\delta T_{m 5}
\end{array}\right]=\left[\begin{array}{ll}
0.5408 & 0.4592 \\
0.0491 & 0.9509
\end{array}\right]\left[\begin{array}{l}
\delta T_{m 2} \\
\delta T_{C 1}^{s}
\end{array}\right] } \\
+\left[\begin{array}{ll}
0.2809 & -0.0009 \\
0.0090 & -0.0041
\end{array}\right]\left[\begin{array}{l}
\delta M c p_{H 2} \\
\delta M c p_{C 1}
\end{array}\right]
\end{aligned}
$$

Table 6. Input data for E-5

\begin{tabular}{|c|c|c|c|}
\hline Stream & $T_{\text {in }}\left({ }^{\circ} \mathrm{C}\right)$ & $T_{\text {out }}\left({ }^{\circ} \mathrm{C}\right)$ & $\mathrm{Mcp}\left(K w^{\circ} K^{-1}\right)$ \\
\hline $\mathrm{H} 3$ & 125 & 67.18 & 23.9 \\
\hline $\mathrm{C} 1$ & 47.75 & 50.683 & 466.7 \\
\hline
\end{tabular}

$$
\begin{aligned}
{\left[\begin{array}{l}
\delta T_{H 3}^{t} \\
\delta T_{m 3}
\end{array}\right]=\left[\begin{array}{ll}
0.2515 & 0.7485 \\
0.0380 & 0.9620
\end{array}\right]\left[\begin{array}{l}
\delta T_{H 3}^{s} \\
\delta T_{m 4}
\end{array}\right] } \\
+\left[\begin{array}{ll}
1.5139 & -0.0024 \\
0.0459 & -0.0062
\end{array}\right]\left[\begin{array}{l}
\delta M c p_{H 3} \\
\delta M c p_{C 1}
\end{array}\right]
\end{aligned}
$$

Table 7. Input data for E-6

\begin{tabular}{|c|c|c|c|}
\hline Stream & $T_{\text {in }}\left({ }^{\circ} \mathrm{C}\right)$ & $T_{\text {out }}\left({ }^{\circ} \mathrm{C}\right)$ & $\mathrm{Mcp}\left(K w^{\circ} K^{-1}\right)$ \\
\hline $\mathrm{H} 5$ & 90 & 86 & 1500 \\
\hline $\mathrm{C} 2$ & 55 & 65 & 600 \\
\hline
\end{tabular}$$
\left[\begin{array}{l}
\delta T_{m 1} \\
\delta T_{m 4} \\
\delta T_{H 1}^{t} \\
\delta T_{m 6} \\
\delta T_{m 2} \\
\delta T_{C 1}^{t} \\
\delta T_{H 2}^{t} \\
\delta T_{m 5} \\
\delta T_{H 3}^{t} \\
\delta T_{m 3} \\
\delta T_{H 5}^{t} \\
\delta T_{C 2}^{t} \\
\delta T_{H 6}^{t} \\
\delta T_{C 4}^{t}
\end{array}\right]
$$

$=\left[\begin{array}{cccc}0.5999 & 0.4001 & 0 & 0 \\ 0.1340 & 0.8660 & 0 & 0 \\ 0 & 0 & 0.6941 & 0.3059 \\ 0 & 0 & 0.5882 & 0.1180 \\ 0 & 0 & 0 & 0 \\ 0 & 0 & 0 & 0 \\ 0 & 0 & 0 & 0 \\ 0 & 0 & 0 & 0 \\ 0 & 0 & 0 & 0 \\ 0 & 0 & 0 & 0 \\ 0 & 0 & 0 & 0 \\ 0 & 0 & 0 & 0 \\ 0 & 0 & 0 & 0 \\ 0 & 0 & 0 & 0\end{array}\right.$

$$
\begin{aligned}
{\left[\begin{array}{l}
\delta T_{H 5}^{t} \\
\delta T_{C 2}^{t}
\end{array}\right]=\left[\begin{array}{ll}
0.8857 & 0.1143 \\
0.2857 & 0.7143
\end{array}\right]\left[\begin{array}{l}
\delta T_{H 5}^{s} \\
\delta T_{C 2}^{s}
\end{array}\right] } \\
+\left[\begin{array}{ll}
0.0025 & -0.0009 \\
0.0003 & -0.0143
\end{array}\right]\left[\begin{array}{l}
\delta M c p_{H 5} \\
\delta M c p_{C 2}
\end{array}\right]
\end{aligned}
$$

Table 8. Input data for E-7

\begin{tabular}{|c|c|c|c|}
\hline Stream & $T_{\text {in }}\left({ }^{\circ} \mathrm{C}\right)$ & $T_{\text {out }}\left({ }^{\circ} \mathrm{C}\right)$ & $\mathrm{Mcp}\left(K w^{\circ} K^{-1}\right)$ \\
\hline H6 & 225 & 75 & 50 \\
\hline $\mathrm{C} 4$ & 43.96 & 136.23 & 81.3 \\
\hline
\end{tabular}

$$
\begin{aligned}
{\left[\begin{array}{l}
\delta T_{H 6}^{t} \\
\delta T_{C 4}^{t}
\end{array}\right]=\left[\begin{array}{ll}
0.1714 & 0.8286 \\
0.5096 & 0.4904
\end{array}\right]\left[\begin{array}{l}
\delta T_{H 6}^{S} \\
\delta T_{m 2}
\end{array}\right] } \\
+\left[\begin{array}{ll}
1.7570 & -0.1701 \\
0.7644 & -0.8456
\end{array}\right]\left[\begin{array}{l}
\delta M c p_{H 6} \\
\delta M c p_{C 4}
\end{array}\right]
\end{aligned}
$$

\subsection{System based disturbance propagation}

\section{model}

Using equations 9 through 12 and lumping equations (19 to 25 ) the model in equation- 8 can be obtained in equation- 26 as follows: 


\begin{tabular}{|c|c|c|c|c|c|c|c|c|c|c|c|c|c|c|}
\hline$[0.0881$ & -0.0025 & 0 & 0 & 0 & 0 & 0 & 0 & 0 & 0 & 0 & 0 & 0 & 0 & {$\left[\delta M c p_{H 1}\right]$} \\
\hline 0.0074 & -0.0115 & 0 & 0 & 0 & 0 & 0 & 0 & 0 & 0 & 0 & 0 & 0 & 0 & $\delta M c p_{C 1}$ \\
\hline 0 & 0 & 0.0958 & -0.0639 & 0 & 0 & 0 & 0 & 0 & 0 & 0 & 0 & 0 & 0 & $\delta M c p_{H 1}$ \\
\hline 0 & 0 & 0.0332 & -0.2950 & 0 & 0 & 0 & 0 & 0 & 0 & 0 & 0 & 0 & 0 & $\delta M c p_{C 4}$ \\
\hline 0 & 0 & 0 & 0 & 0.5717 & -0.0027 & 0 & 0 & 0 & 0 & 0 & 0 & 0 & 0 & $\delta M c p_{H 2}$ \\
\hline 0 & 0 & 0 & 0 & 0.0251 & -0.0090 & 0 & 0 & 0 & 0 & 0 & 0 & 0 & 0 & $\delta M c p_{C 1}$ \\
\hline 0 & 0 & 0 & 0 & 0 & 0 & 0.2809 & -0.0009 & 0 & 0 & 0 & 0 & 0 & 0 & $\delta M c p_{H 2}$ \\
\hline 0 & 0 & 0 & 0 & 0 & 0 & 0.0090 & -0.0041 & 0 & 0 & 0 & 0 & 0 & 0 & $\delta M c p_{C 1}$ \\
\hline 0 & 0 & 0 & 0 & 0 & 0 & 0 & 0 & 1.5139 & -0.0024 & 0 & 0 & 0 & 0 & $\delta M c p_{H 3}$ \\
\hline 0 & 0 & 0 & 0 & 0 & 0 & 0 & 0 & 0.0459 & -0.0062 & 0 & 0 & 0 & 0 & $\delta M c p_{C 1}$ \\
\hline 0 & 0 & 0 & 0 & 0 & 0 & 0 & 0 & 0 & 0 & 0.0025 & -0.0009 & 0 & 0 & $\delta M c p_{H 5}$ \\
\hline 0 & 0 & 0 & 0 & 0 & 0 & 0 & 0 & 0 & 0 & 0.0003 & -0.0143 & 0 & 0 & $\delta M c p_{C 2}$ \\
\hline 0 & 0 & 0 & 0 & 0 & 0 & 0 & 0 & 0 & 0 & 0 & 0 & 1.7570 & -0.4701 & $\delta M c p_{H 6}$ \\
\hline 0 & 0 & 0 & 0 & 0 & 0 & 0 & 0 & 0 & 0 & 0 & 0 & 0.7644 & -0.8456 & {$\left[\begin{array}{l}\delta p p \\
\delta\end{array}\right]$} \\
\hline
\end{tabular}

\section{(Equation-26)}

After equation-26 is constructed and by applying equations (15 to 17) and using system construction matrices V1, V2 and
V3 yields system disturbance propagation model in equation14 as follows: $\left[\begin{array}{l}\delta T_{H 1}^{t} \\ \delta T_{H 2}^{t} \\ \delta T_{H 3}^{t} \\ \delta T_{H 4}^{t} \\ \delta T_{H 5}^{t} \\ \delta T_{H 6}^{t} \\ \delta T_{C 1}^{t} \\ \delta T_{C 2}^{t} \\ \delta T_{C 3}^{t} \\ \delta T_{C 4}^{t}\end{array}\right]=\left[\begin{array}{cccccc}0.6941 & 0 & 0 & 0 & 0 & 0 \\ 0.1849 & 0.2264 & 0 & 0 & 0 & 0 \\ 0.0126 & 0.0154 & 0.2515 & 0 & 0 & 0 \\ 0 & 0 & 0 & 0 & 0 & 0 \\ 0 & 0 & 0 & 0 & 0.8857 & 0 \\ 0.0134 & 0.0164 & 0.0315 & 0 & 0 & 0.1714 \\ 0.5516 & 0.0623 & 0 & 0 & 0 & 0 \\ 0 & 0 & 0 & 0 & 0.2857 & 0 \\ 0 & 0 & 0 & 0 & 0 & 0 \\ 0.0079 & 0.0097 & 0.0186 & 0 & 0 & 0.5096\end{array}\right.$

$+\left[\begin{array}{ccccc}0.1892 & 0 & 0 & 0 & 0 \\ 0.0353 & 0.5901 & 0 & 0 & 0 \\ 0.0024 & 0.0277 & 1.5139 & 0 & \\ 0 & 0 & 0 & 0 & \\ 0 & 0 & 0 & 0 & 0.00 \\ 0.0026 & 0.0295 & 0.0381 & 0 & \\ 0.1054 & 0.0251 & 0 & 0 & \\ 0 & 0 & 0 & 0 & 0.00 \\ 0 & 0 & 0 & 0 & 0 \\ 0.0015 & 0.0175 & 0.0225 & 0 & 0\end{array}\right.$

As equation-27 is constructed any disturbance occurs in the HEN can be evaluated. Table 9 summarize the results of the disturbance expected and its effect on streams target temperatures.

Table 9.Represent the deviation in the streams target temperatures.

\begin{tabular}{|c|c|c|c|c|}
\hline $\begin{array}{c}\text { Streams } \\
\text { target } \\
\text { temp. }\end{array}$ & $\begin{array}{c}\delta T_{H 1}^{S} \\
5^{\circ} \mathrm{C}\end{array}$ & $\begin{array}{c}\delta T_{H 2}^{S} \\
-10^{\circ} \mathrm{C}\end{array}$ & $\begin{array}{c}\delta T_{H 3}^{S} \\
+5^{\circ} \mathrm{C}\end{array}$ & $\begin{array}{c}\delta M c p_{C 1} \\
2 \%\end{array}$ \\
\hline$\delta T_{H 1}^{t}$ & 3.4707 & 0 & 0 & -0.0978 \\
\hline$\delta T_{H 2}^{t}$ & 0.9246 & -2.2639 & 0 & -0.0486 \\
\hline$\delta T_{H 3}^{t}$ & 0.0629 & -0.1539 & 1.2576 & -0.0531 \\
\hline$\delta T_{H 4}^{t}$ & 0 & 0 & 0 & 0 \\
\hline$\delta T_{H 5}^{t}$ & 0 & 0 & 0 & 0 \\
\hline$\delta T_{H 6}^{t}$ & 0.0669 & -0.1639 & 0.1573 & -0.0808 \\
\hline$\delta T_{C 1}^{t}$ & 2.7578 & -0.6228 & 0 & -0.1613 \\
\hline
\end{tabular}

$\left.\begin{array}{cccc}0 & 0 & 0 & 0.3059 \\ 0.4592 & 0 & 0 & 0.1295 \\ 0.7117 & 0 & 0 & 0.0088 \\ 0 & 0 & 0 & 0 \\ 0 & 0.1143 & 0 & 0 \\ 0.7580 & 0 & 0 & 0.0094 \\ 0 & 0 & 0 & 0.3862 \\ 0 & 0.7143 & 0 & 0 \\ 0 & 0 & 0 & 0 \\ 0.4486 & 0 & 0 & 0.0055\end{array}\right]\left[\begin{array}{l}\delta T_{H 1}^{S} \\ \delta T_{H 2}^{S} \\ \delta T_{H 3}^{S} \\ \delta T_{H 4}^{s} \\ \delta T_{H 5}^{s} \\ \delta T_{H 6}^{s} \\ \delta T_{C 1}^{s} \\ \delta T_{C 2}^{s} \\ \delta T_{C 3}^{s} \\ \delta T_{C 4}^{s}\end{array}\right]$

$$
\left.\begin{array}{ccccc}
0 & -0.0105 & 0 & 0 & -0.0639 \\
0 & -0.0052 & 0 & 0 & -0.0928 \\
0 & -0.0057 & 0 & 0 & -0.0063 \\
0 & 0 & 0 & 0 & 0 \\
0 & 0 & -0.0010 & 0 & 0 \\
1.7570 & -0.0087 & 0 & 0 & -0.4768 \\
0 & -0.0173 & 0 & 0 & -0.2767 \\
0 & 0 & -0.0143 & 0 & 0 \\
0 & 0 & 0 & 0 & 0 \\
0.7644 & -0.0051 & 0 & 0 & -0.8495
\end{array}\right]\left[\begin{array}{l}
\delta M c p_{H 1} \\
\delta M c p_{H 2} \\
\delta M c p_{H 3} \\
\delta M c p_{H 4} \\
\delta M c p_{H 5} \\
\delta M c p_{H 6} \\
\delta M c p_{C 1} \\
\delta M c p_{C 2} \\
\delta M c p_{C 3} \\
\delta M c p_{C 4}
\end{array}\right]
$$

\begin{tabular}{|c|c|c|c|c|}
\hline$\delta T_{C 2}^{t}$ & 0 & 0 & 0 & 0 \\
\hline$\delta T_{C 3}^{t}$ & 0 & 0 & 0 & 0 \\
\hline$\delta T_{C 4}^{t}$ & 0.0396 & -0.0970 & 0.0931 & -0.0473 \\
\hline
\end{tabular}

\section{CONCLUSION}

In this paper a linear model was constructed to evaluate the disturbance in streams target temperatures. Each exchanger in the network is represented by a set of algebraic equation with neglect high order differentiation terms. The model can be used to estimate the maximum deviation of system outputs. The determination of heat exchanger network target temperature can be carried out in different ways. Several equations can be applied to find outlet temperatures of a network. It is easy to use computationally efficient and particularly helpful in analyzing integrated process systems where disturbance propagation is always a major concern. The efficacy of using the model demonstrated by solving practical industrial problems demonstrate the applicability of the model in process analysis and improvement. 


\section{REFERENCES}

[1] Picon-Nunez, M. and Polley, G. T., 1995, Determination of the steady state response of heat exchanger networks without simulation, Trans IChemE, Part A, Chem Eng Res Des, 13(A1): 49-58.

[2] Kotjabasakis, E.; Linnhoff, B.,1986., Sensitivity Tables for the Design of Flexible Process (I) - How Much Contingency in Heat Exchanger Networks Is CostEffective? Chem. Eng. Res. 64, 197.

[3] Ratnam, R. and Patwardhan, V S., 1991, Sensitivity analysis for heat exchanger networks, Chem Eng Sci, 46(2): 451-458.

[4] Kern, D. Q., 1950, Process Heat Transfer (McGraw Hill, New York, USA).

[5] Li, G. Q.; Hua, B.; Liu, B. L.; Wu, G. R.,1994., The Study for Flexibility Analysis Method in Heat Exchanger
Network. Proc. PSE p-407.

[6] Yang, Y. H., Gong, J. P and Huang, Y. L., 1996, A simplified system model for rapid evaluation of disturbance propagation through a heat exchanger network, Ind Eng Chem Res, 35(12): 4550-4558.

[7] P.J HEGGS and F. VIZCAINO.,2002., A rigorous model for evaluation of disturbance propagation through heat exchanger networks, Trans IChemE, Vol 80, Part A.

[8] Ahmad, S. (1985)., Heat exchanger networks., cost tradeoffs in energy and capital, Ph.D. thesis. UK., UMIST Manchester.

[9] Krishna M.yerramsetty and C.V.S. Murty.,2008, Synthesis of cost-optimal heat exchanger networks using differential evolution., Computer and chemical engineering 32,861-1876. 